Voix et Images

voixetimages

\title{
Le récit télévisuel et son écriture
}

\section{Jacques La Mothe}

Volume 9, numéro 1, automne 1983

Guy Dufresne

URI : https://id.erudit.org/iderudit/200418ar

DOI : https://doi.org/10.7202/200418ar

Aller au sommaire du numéro

Éditeur(s)

Université du Québec à Montréal

ISSN

0318-9201 (imprimé)

1705-933X (numérique)

Découvrir la revue

Citer cet article

La Mothe, J. (1983). Le récit télévisuel et son écriture. Voix et Images, 9(1), 7-27. https://doi.org/10.7202/200418ar d'utilisation que vous pouvez consulter en ligne.

https://apropos.erudit.org/fr/usagers/politique-dutilisation/ 


\title{
Le récit télévisuel et son écriture
}

\author{
par Jacques La Mothe, Univerșité du Québec à Montréal
}

N.D.L.R.

Le texte qui suit ne concerne pas Guy Dufresne. I/ nous a cependant paru suffisamment instructif pour être inséré dans ce dossier qui traite en grande partie de l'écriture télévisuelle.

Dès ses débuts, la télévision est apparue.comme un prodigieux moyen de création artistique et, que ce soit en Amérique ou en Europe, plusieurs théoriciens s'inspirant de la pratique de ce média ont tenté de définir les paramètres de ce nouveau langage et de codifier la production des récits télévisuels qu'il s'agisse de la narration, de la manière de raconter, ou de la fiction, de ce qui est raconté. En effet, si pour les uns la télévision n'était qu'un simple véhicule et n'encourageait pas ou très peu d'espoir au niveau artistique, au contraire pour d'autres comme Renato May, Angelo d'Alessandro, Marcel l'Herbier, André Brincourt, André Veinstein, la télévision dans certaines conditions favorisait la recherche d'un langage et d'une forme d'art à la fois nouveaux et spécifiques.

Mais en général, du point de vùe de ceux qui en réalisent les émissions, la période qui semble avoir été la plus fructueuse, voire la plus exaltante pour certains, semble se situer dans plusieurs pays entre 1955 et 1965 . Avant 1955, il s'agissait beaucoup plus d'une période d'apprentissage pour les employés de chaque réseau; pour les spectateurs, une phase d'acquisition des appareils récepteurs, et enfin pour tous les intéressés, un moment d'émerveillement face à ce nouveau phénomène. Mais il semble aussi que; depuis 1965, persiste une certaine stagnation et ce, non pas au niveau de la production, mais plutôt à celui des formes et des idées, toujours les mêmes, ressassées jusqu'à leur cristallisation.

Par ailleurs, du point de vue du spectateur, le téléviseur est la source de diffusion d'un flux ininterrompu de récits, et si certains codes utilisés à la télévision sont plus ou moins spécifiques à ce média, le plus intéressant n'est pas tant l'étude de leur spécificité que celle de leur mise en jeu; nous allons. 
donc ici tenter de cerner les paramètres du récit télévisuel dans ce qu'il a d'inventif, et à travers cela, de discerner les formes narratives en présence.

\section{Le direct comme forme}

Pour dissiper un malentendu selon lequel le direct est un genre d'émission, mentionnons au préalable que le passage direct de la scène au signal électrique dans la caméra n'est que l'un des divers procédés d'enregistrement de l'image que permet la télévision, les autres étant :

- l'enregistrement optique sur film d'une image électronique grâce au «Kinescope»; c'est un procédé maintenant désuet;

- le film avec transformation de l'image photographique en image électronique dans le télécinéma;

- l'enregistrement magnétique sur magnétoscope : enregistrement du signal électrique fourni par la caméra sur bande magnétique.

Par contre, la définition d' «enregistrement» accordée à la prise de vue en direct laisse à désirer puisque la diffusion instantanée n'implique pas nécessairement un enregistrement que l'on peut conserver : en réalité, une émission dite «en direct» est transmise sans support matériel. Il nous faudra donc distinguer la réalité du direct et la forme du direct puisqu'il existe des émissions filmées qui adoptent la morphologie du direct.

Une partie de hockey ou un match de football seront d'habitude transmis en direct et simultanément enregistrés sur pellicule; le soir ou le lendemain, des extraits de cet enregistrement seront diffusés aux informations sportives par exemple, et s'il s'agit d'une partie ou d'un match intéressant, important, voire stratégique, un montage sélectif supprimant les temps morts pourra être effectué en vue d'une diffusion ultérieure. Dans le premier cas, il s'agit d'une émission en direct, mais dans les cas subséquents il ne s'agit plus de direct mais de formes dérivées qui adoptent en partie la même structure que les émissions directes.

Une seconde remarque que l'on peut faire au sujet de la diffusion instantanée est qu'il y a en quelque sorte contradiction entre les possibilités narratives qu'offre l'emploi de cette technique, possibilités que l'on tend toutefois à éviter. Si le direct, mettant l'accent sur l'indétermination, utilisait la succession brute d'événements, sans intrigue univoque mais en conservant leur champ de possibilités, en orientant le spectateur vers d'autres perspectives, on pourrait en arriver à des oeuvres ayant une «ouverture» telle que peuvent en pratiquer certains types de communication artistique, la musique, le roman ou le cinéma, par exemple.

Ce serait toutefois oublier la nature du procédé utilisé, sa destination sociale, les dispositions psychologiques du spectateur, son conditionnement en attente face à un événement qui peut ou va se produire, son attente d'un produit déterminé et son aspiration à un profond besoin d'intrigue; ce serait 
aussi oublier les tendances acquises du réalisateur et surtout, le conditionnement de ses structures de narration. «Justement parce qu'elle a pour objet la vie comme hasard, la prise de vue en direct cherche à la dominer en recourant à un mode d'organisation traditionnel, de type aristotélicien, régi par ces lois de causalité et de nécessité qui sont, en définitive, les lois mêmes de la vraisemblance». ${ }^{1}$

La prise de vue et la mise en ondes simultanées à l'événement qui se déroule supposent une interprétation de l'événement, puisqu'il y a un premier choix quant à l'emplacement des microphones et des caméras, suivi d'un second choix, à savoir quelle image des trois habituellement reçues en régie, sera diffusée. Ce montage séra contemporain de l'événement et les réflexes et l'intuition prendront une certaine importance dans sa réalisation; on peut dire que, jusqu'à un certain point, il s'agit d'un montage improvisé bien que la plupart des événements présentés en direct laissent une très faible marge d'interprétation, une part en réalité bien minime de choix. $\mathrm{Si}$ les événements extraordinaires, du couronnement de la Reine d'Angleterre au premier homme sur la lune, sont préparés dans les moindres détails, la partie de hockey ou le match de football sont eux-mêmes régis par des règles très strictes dans un lieu et une durée bien déterminés; le centre d'intérêt, ce sont les déplacements de la rondelle ou du ballon et les différentes tactiques utilisées par les joueurs jusqu'au but, ou au but manqué : les limites de l'invention sont donc considérablement réduites et partant, les possibilités artistiques que pourrait of frir cette forme de traitement.

Le réalisateur, comme le fait remarquer Eco, se trouve dans la position du conteur, construisant «une narration conforme à un principe élémentaire de cohérence et réalisée au moment même où on la conçoit,... ce qu'on pourrait appeler un récit impromptu». ${ }^{2} \mathrm{Le}$ récit moulé sur une série d'événements plus ou moins autonomes (certains cependant font partie d'ensembles ayant une cohérence préalable, la partie de hockey par exemple possède ses structures de base : règles du jeu, groupes de protagonistes, etc.) nécessitera un fil narratif et le réalisateur, pour conserver l'unité de son récit parmi un certain nombre d'intrigues possibles, va isoler un événement central et l'enrichir, l'illustrer par les événements significatifs bien qu'inessentiels, marginaux, dont il disposera. En réalité, il va devoir «inventer l'événement au moment même où il se produit, et l'inventer tel qu'effectivement il se produit». ${ }^{3}$ Ainsi, avec cet événement synthétique, la question : «Est-ce vrai?» est moins importante que celle-ci : «Y a-t-il matière à nouvelle?»

1. ECO, Umberto, in l'OEuvre ouverte, Paris, Éditions du Seuil, coll. Pierres Vives, 1965, traduit de l'édition italienne chez Bompiani, Milan, 1962, Opera Aperta, p. 160.

2. Idem, p. 151 .

3. Idem, p. 156. 
Si l'«ouverture» s'est révélée être une forme à laquelle la télévision en direct est plutôt réfractaire, il serait intéressant de jeter un coup d'œil aux formes générées ou suscitées par cette technique ou, pourrait-on dire aussi bien, aux formes par lesquelles le direct s'est exprimé avec le plus d'aisance. Mentionnons tout d'abord que le temps du direct tend à se confondre avec le temps réel et que, par conséquent, l'action peut parfois être alourdie par les temps morts que la réalité comporte : à la suite d'un certain cinéma qui nous avait habitués à un temps plein, actif, les utilisateurs du direct ont remédié partiellement à cet état de choses en effectuant des reprises au moyen du téléciné. D'autre part, l'élément verbal appartient au langage parlé et n'a pas été écrit auparavant. Enfin, l'image du direct n'est pas nécessairement parfaite du point de vue technique, et n'a pas de frontières strictes puisque peuvent y apparaître, parfois involontairement certes, les moyens de production de l'image. On a aussi pu remarquer que, jusque vers 1960 , les plans tendaient à être plus longs qu'au cinéma et que le direct utilisait plus de plans rapprochés et de plans américains que le cinéma.

Par une sorte de circulation des formes ou de mimétisme entre les différents media, l'expression propre au direct n'a pas été longtemps du domaine exclusif de la télévision. Le mode de travail du direct avait déjà hérité de celui que le reportage filmé avait prodigieusement développé durant la seconde guerre mondiale : le cinéma allait maintenant être bouleversé par l'apparition de la télévision. Autour des années 60 , il tend à assimiler les formes nouvelles que lui suggère le direct, entre autres l'ouvre d'Antonioni (surtout «l'Avventura», «la Notte», «l'Eclisse», «il Deserto rosso»), certains films de la Nouvelle Vague en France ( $« \grave{A}$ bout de souffle», par exemple, où la durée de l'action coïncide avec celle de la projection), et bien entendu le "cinéma-vérité» (l'expression est d'Edgar Morin) ${ }^{4}$ ou «cinéma direct» illustrant bien la répercussion de la télévision dans «son expression la plus pure» selon certains, c'est-à-dire le direct, sur le septième art.

Avec le développement du film et du magnétoscope, le direct qui longtemps a constitué la substance de la spécificité télévisuelle, maintenant utilisé au minimum de ses possibilités, est devenu strictement véhiculaire et s'est révélé, à l'usage, bien peu créateur : s'il est opposé au téléfilm, il apparaît comme refuge du traditionnel (informations, quiz, retransmissions sportives). Est-ce à cause des risques qu'il comporte, de son aspect incontrôlable devant des faits qui sortent des limites du prévisible et qui lui donnent une allure bâclée? Toutefois, bien plus que dans sa technique, il semble que son importance se situerait plutôt dans la conscience qu'en a le spectateur.

4. MORIN, Edgar, "Pour un nouveau cinéma-vérité̀, in France Observateur, nº 506, 14 janvier 1960. 


\section{Une écriture codifiée}

Aux États-Unis, c'est autour de plusieurs émissions de «télé-théâtre» comme Kraft-Theatre, Studio One, Playhouse 90 que sont apparus un certain nombre d'auteurs dont les oeuvres ont marqué jusque vers 1955 la production télévisuelle : entre autres Reginald Rose avec "Crime in the Streets», "Twelve Angry Men», "The Sacco and Vanzetti Story»; Robert Alan Arthur avec "A Man is Ten Feet Fall», "Spring Reunion»; Rod Sterling avec «Patterns» et «The Rack» et surtout Paddy Chayefsky avec «Marty», "The Wedding Party», "Middle of the Night», "The Bachelor Party» et plus tard Gore Vidal avec "Visit to a Small Planet». Venus pour la' plupart du théâtre et influencés par Tennessee Williams, ils s'inspirent des premiers théoriciens de la télévision pour qui les particularités de ce média étaient l'immédiateTé, la spontanéité, l'actualité et l'intimité.

Les différents réseaux de télévision ont tout d'abord été fortement influencés par le style d'expression développé par les Américains, caractérisé par une économie de moyens au niveau du nombre de décors et de personnages, par la réalisation en studio d'œuvres d'une durée limitée à une heure et dont le sujet habituellement réaliste et contemporain est traité de manière intimiste, par la tentative enfin de rompre avec le cinéma bien que bon nombre de ces émissions de fiction dramatique aient été reprises au cinéma ou encore au théâtre : "Twelve Angry Men» par exemple, mais aussi certaines oeuvres de Guy Dufresne et de Marcel Dubé. On note aussi un certain rapprochement avec les techniques et les thèmes du documentaire.

Il semble que le point de départ commun, mais aux voies divergentes, de la recherche d'une expression propre à la télévision en France et en Angleterre fut le congrès de Gardone en Italie, qui eut lieu en 1956, möment où la production de Rose et de Chayefsky avait acquis une importance considérable, moment aussi où les États-Unis et l'Italie avaient déjà adapté des ouvres littéraires, nouvelles ou romans, pour la télévision et s'étaient en quelque sorte, avec la B.B.C., affranchies du théâtre, ce que n'avait pas encore fait la télévision française si bien qu'en France, à la suite de ce congrès, on abandonna progressivement le vidéo et les studios pour se rapprocher du film alors qu'en Angleterre on faisait le contraire!

En France, c'est avec Jean d'Arcy et Albert Ollivier que l'on élabora ce que l'on peut appeler une "politique des textes", si l'on entend par là une étude des conditions de production et d'expression qu'exigeait la télévision, et l'édiction de règles tentant de cerner la. «spécificité» de l'expression télévisuelle. Entre 1954 où la télévision française produisait 81 dramatiques et 1967 où elle en produit 203, on peut mesurer l'évolution qui est apparue à ce niveau.

En Angleterre, avec Michael Barry puis Sidney Newman et Gerald Savoy, c'est plutôt une «politique d'auteurs» qui se développe avec le «story-editor» qui s'occupe de conseiller les écrivains, d'entretenir de 
bonnes relations avec eux et de leur négocier des cachets convenables : c'est ainsi que la télévision anglaise peut produire jusqu'à 700 dramatiques par année. Par ce moyen, et contrairement à la France qui s'est constitué une banque d'auteurs et d'adaptateurs ${ }^{5}$ qui acceptent les règles de l'écriture télévisuelle, l'Angleterre peut se permettre de révéler tous les ans trente auteurs inconnus. Parmi les révélations les plus brillantes figurent John Arden et Harold Pinter («The Collection», "The Lover»). L'Angleterre peut puiser parmi 1,400 écrivains qui ont déjà travaillé pour la télévision. De plus, il est admis que la grammaire télévisuelle procède essentiellement de celle du cinéma ${ }^{6}$, ce qui évite toutes les théories sur la "spécificité» télévisuelle!

Bien que l'écriture à la télévision ne soit pas nécessairement condamnée à l'académisme et à l'adaptation littéraire, le fait de l'astreindre à des règles d'ordre "esthétique» qui parfois atteignent sinon un certain cynisme, du moins une naïveté certaine, risque d'exclure toute autonomie de la composition. Si les contingences socio-économico-politiques du marché du livre n'ont que peu d'influence sur le contenu des volumes, au cinéma on peut en ressentir les pressions d'une manière plus précise et à la télévision, on peut alors vraiment parler de contraintes puisque son principal souci est de rendre sa production conforme à l'idéologie en place et de ressembler à la narration la plus commerciale possible, «si bien que les auteurs de télévision sont automatiquement amenés à faire le produit que la télévision demande». ${ }^{7}$

\section{Aux États-Unis...}

À l'origine écrivain de théâtre, Chayefsky, dans la préface de ses ceuvres pour la télévision, définit brièvement mais avec assez de précisions les caractéristiques propres à l'auteur et à la télévision américaine vers 1955 .

L'auteur de télévision doit s'adapter aux limitations précises et innombrables de son instrument et aborder son travail de rédaction sous un angle particulier. Il doit par exemple tenir compte que le montage électronique consiste dans le passage d'une caméra à une autre, tandis qu'au cinéma, le monteur taille ici et là dans la matière filmée.

Selon lui, on ne peut pas faire évoluer avec aisance plus de quatre personnes à la fois sur l'écran; contrairement au thêtre où il y a une convention de l'impressionnisme, à la télévision où le spectateur attend qu'on lui montre le réel, il est périlleux de faire jouer une foule par dix personnes.

5. ANDREY, Roger, "La télévision en quête d'auteurs», in la Technique et l'Exploitation cinématographique, no 299, septembre 1968.

6. DESMOND, Davis, The Grammar of Television Production, Barrie \& Rocliff, 1969.

7. ROBBE-GRILLET, Alain, in Robbe-Grillet/Colloque de Cerisy, vol. 2, coll. 10/18,Union Générale d'Éditions, 1972, p. 425. 
Pour rendre la production «plus satisfaisante», il est préférable de lier les scènes au moyen du fondu et de réserver les coupes aux moments de fortes tensions dramatiques; pour les scènes au téléphone, Chayefsky préfère, plutôt que la coupe à chaque ligne de dialogue, la division de la scène en deux parties égales.

Hors ces considérations «esthétiques», il insiste surtout sur les limites qu'impose la télévision à l'auteur, dont la principale est le temps : un drame d'une heure ne doit durer que cinquante-trois minutes, cela implique que la télévision ne s'accommode pas de textures dramatiques fortement tramées, mais plutôt d'une intrigue principale, que peuvent compléter parfois une, jamais plus de deux intrigues secondaires.

L'histoire à tiroirs, c'est-à-dire une histoire où plusieurs sujets secondaires s'enroulent autour d'un incident central sans contribuer à le faire valoir ou à lui faire prendre forme, mais où, précisément, il y a une progression dramatique simultanée des divers sujets, ce qui entraîne le passage d'une histoire à l'autre, cela la télévision ne l'accepte pas. La structure du script, chacune des histoires étant toujours en mouvement, ne propose ni premier ni second acte; or ceux-ci sont nécessaires pour introduire la publicité : «La présence même de la publicité indique les limitations les plus générales et les plus strictes de la technique de la télévision. La télévision est essentiellement un instrument de publicité et non un moyen de divertissement. Les agences de publicité ne se soucient que de vendre les produits de leurs clients et elles ne désirent pas des drames susceptibles de troubler les acheteurs éventuels. Ce qui limite considérablement le choix des sujets».$^{8}$

Les sujets à proscrire sont à ce moment, aux États-Unis, l'adultère, l'avortement, les valeurs sociales de l'époque, les controverses politiques et même les histoires à dénouement tragique, en d'autres mots tout ce qui traite sans complaisance des réalités adultes.

Il est intéressant de noter que ces thèmes, à l'exception des controverses politiques, constituent à peu près le relevé ou l'inventaire des sujets qu'allaient traiter à peu près à la même époque Marcel Bluwal et Marcel Moussy à l'O.R.T.F., alors exempte de publicité, dans la série dramatique «Si c'était vous». Tabous culturels américains ou censure commerciale?

Le genre de sujet qui intéresse la télévision serait plutôt le «drame intime», c'est-à-dire des études minutieusement détaillées des petits moments de l'existence caractérisées par «la volonté de creuser sous la surface de la vie pour y découvrir les vérités fondamentales des rapports entre les humains». 9

8. CHAYEFSKY, Paddy, «L'écrivain de télévision», in Les Cahiers du Cinéma, $\mathrm{n}^{\circ} \mathbf{9 0}$, décembre 1958, Tome XV, traduction de Louis Marcorelles.

9. FRANK, André, "Deuxième entretien...», in Les Cahiers Renaud-Barrault, no 43, mars 1963, p. 114, Paris, Julliard. 


\section{en France...}

Trois idées directrices allaient animer le travail relatif à l'écriture télévisuelle que se proposait de mener à bien André Frank (chef du service des dramatiques de télévision à l'O.R.T.F. jusqu'en 1970) :

- la proposition d'un domaine insuffisamment exploré : celui du récit;

- la conquête progressive de genres nouveaux;

- l'adhésion à des idées que l'état même de la technique imposait.

Frank; avec, entre autres, la collaboration de Mme Jacqueline Capet et de MM. Lucien Jolivald et Robert Vosse, va essayer d'établir des principes parfois définis comme des lois qui ne sont «que des lois techniques et non des lois esthétiques» ${ }^{10}$, parfois comme «la définition des bases d'une Esthétique du contenant et du contenu» ${ }^{11}$, mais aussi plus prudemment, comme des «indications précaires». ${ }^{12}$

Il établit en premier lieu une série de comparaisons entre le théâtre, le cinéma et la télévision à partir d'éléments communs considérés sous l'angle de leur efficacité :

\section{Oeuvres présentées} sur une scène

Unité du spectacle considérée dans son mouvement et ses mouvements.

Affirmation d'un rythme.

Dialogue.

Présence et timbre de la voix.

Décor dans son style.

Viságes et jeux:

Les lumières dans leur possibilité de changements et leur pouvoir de création d'une atmosphère.

\section{Films}

Découpage.

Mouvement et recherche d'un rythme.

Décor dans sa complexité.

Lumières et images.

Visages.

Dialogues.
Émissions

dramatiques

de télévision

Dialogues et recherches d'un rythme.

Cheminement du récit. Lumières: choix des images.

Les visages: présence du comédien: puissance d'expression du regard; importance et stricte concordance des âges.

Mouvements.

Décors dans leur dépouillement; accessoires dans leur exigence essentielle.

10. FRANK, André, "Les seules voies", in la Dramatique et la Télévision, brochure ronéotypée de l'O.R.T.F., no T/1527 B, p. 4

11. FRANK, André, «Notes provisoires à l'intention des auteurs, scénaristes et dialoguistes d'émissions dramatiques de télévision», in Études cinématographiques, nos $16-17,2^{e}$ trimestre, 1962.

12. FRANK, André, «Premier entretien sur les-dramatiques», in Les Cahiers RenaudBarrault, no 37 , février 1962, p. 119. 
D'autre part, puisqu'il est nécessaire de repenser certaines œuvres qui n'ont pas été écrites pour la télévision, de les réécrire ou d'en écrire de nouvelles sans pour cela en faire du cinéma ou du théâtre, il a fallu élaborer certains principes de base qui découlent soit d'observations faites à la réception de l'image : dimension de l'écran, distance entre le spectateur et l'écran; soit de l'observation des moyens de production et de leur usage; soit encore de la notion impérative de la durée :

1. L'écran est petit. Dans ses dimensions habituelles, et même si l'on accroît notablement ses proportions, il rend sensibles avant tout la présence humaine, le pouvoị des lumières et des éclairages, grâce à ses images en quelque sorte modelées. Il se prête moins que toute autre forme de spectacle à l'exploitation de la magie des choses. Les objets et les décors n'y ont qu'une efficacité réduite; sauf quand l'action ou le style les réclame;

2. les plans généraux ne devraient être utilisés que dans les cas où l'action les réclame d'une manière impérative. Le gros plan à la télévision est simplement un plan normal;

3. les visages angoissés ou souriants ont une portée particulière, surtout si les propos prennent à témoin le public;

4. les : œuvres qui s'adaptent le mieux à la télévision sont celles qui relèvent de l'intimisme. Ce type d'œuvre y offre le maximum de présence;

5. le petit écran est une loupe; selon l'usage que l'on en fait par rapport aux ouvres, il grandit ou amenuise;

6. on gagne en profondeur, jamais en hauteur, ni en largeur;

7. si l'image reste toujours réduite, le haut-parleur, lui, grandit la voix, d'où la présence des grands textes, dans leur simplicité; pourtant le temps de l'émission reste celui de l'image;

8. le metteur en scène ne réalise pas le texte; il rend plus ou mòins présent un point de départ commun, ici sous forme de sons et là d'images;

9. l'éparpillement est l'ennemi numéro un de l'image; la dramaturgie télévisuelle se montre avide de dépouillement;

10. l'unité compte avant tout : unité entre les intentions du texte, le style du décor, les divers éléments du jeu ou dans le jeü;

11. en matière de décors, il faut distinguer style et stylisation; les chemins naissants de la télévision dramatique ont assimilé parfois l'image avec le décor. Il faudrait maintenant avec plus de justesse distinguer :

a) l'image ramenée au seul visage 
b) l'image limitée au seul groupe

c) l'image façonnée par les lumières, par leur recherche hautement artistique et leur choix des possibles

d) l'image suggérée par les accessoires et par les décors. Un décor surchargé porte au gros plan

12. on accroît les dimensions du petit écran, au besoin, en le fragmentant, jamais en le bourrant;

13. le comique de mouvements ou de mots passe mal;

14. la question du comique semble devoir être reprise en partant des objets et des attitudes;

15. la durée des œuvres doit être appréciée non d'après les critères qui ont cours dans d'autres domaines, mais d'après les temps de fatigue, d'intérêt ou d'attention spécifiques; le petit écran supporte mal les textes trop longs. Une durée d'une heure trente semble être une excellente moyenne.

Par ailleurs, Frank nous fait remarquer que l'écriture dramatique à la télévision est un travail d'équipe; une dramatique s'écrit avec cinq plumes : au premier niveau de synthèse correspond le récit, qui est l'apport du scénariste dont le travail devrait être effectué en concertation avec le réalisateur; suit l'analyse de ce récit avec l'apport du dialoguiste et les recherches du directeur de la photographie; puis la synthèse finale qui appartient au réalisateur.

\begin{tabular}{lll}
$\begin{array}{l}\text { Au récit } \\
\text { scénariste }\end{array}$ & $\begin{array}{l}\text { correspond le premier } \\
\text { projet d'une réalisation } \\
\text { visuelle (réalisateur) }\end{array}$ & $\begin{array}{l}\text { synthèse première et } \\
\text { relativement indéter- } \\
\text { minée }\end{array}$ \\
\hline $\begin{array}{l}\text { Au verbe } \\
\text { dialoguiste }\end{array}$ & $\begin{array}{l}\text { correspond l'image } \\
\text { (directeur de la photo- } \\
\text { graphie) }\end{array}$ & analyse \\
$\begin{array}{l}\text { Il faut considérer le } \\
\text { verbe comme une syn- } \\
\text { thèse poétique primor- } \\
\text { diale, comme logos et } \\
\text { non comme simple } \\
\text { véhicule du sens. }\end{array}$ & \\
\hline $\begin{array}{l}\text { À la phrase } \\
\text { auteur }\end{array}$ & $\begin{array}{l} \\
\text { adaptateur }\end{array}$ & $\begin{array}{l}\text { correspond le rythme } \\
\text { de la réalisation visuelle }\end{array}$ \\
& $\begin{array}{l}\text { (réalisateur-metteur } \\
\text { en scène, décorateur) }\end{array}$ \\
\hline
\end{tabular}


On pourrait ajouter d'autres remarques, par exemple qu'à un geste au théâtre correspond un mouvement du visage à la télévision; ou encore, qu'à la voix au théâtre correspond le regard à la télévision : l'une des grandes règles est donc l'intériorisation. Enfin, Frank considère que les domaines les plus risqués sont ceux qui comportent une part importante de mouvement et de puissance d'éparpillement, ceux qui impliquent le discontinu ou la trop grande diversité.

«Une sorte de force linéaire, de nécessité de rigueur continue, d'affrontement par le seul visage expressif pèse sur le spectacle télévisé comme une hypothèse et aussi à la manière d'un défi». ${ }^{13}$

\section{... en Angleterre...}

En 1960, Donald Wilson, chef du Script Department au Television Service de la B.B.C., et qui sera plus tard producteur de la Dynastie des Forsyte tente de déterminer la fonction de l'écrivain à la télévision, notamment celle de l'auteur dramatique ou «dramaturge» (c'est ainsi que le traducteur interprète le terme de "dramatist», intraduisible en français en tant qu'il s'applique à l'écrivain de T.V.; il s'agit donc de «dramaturge» au sens large du mot ${ }^{14}$ ) dont le travail s'étend du simple sketch jusqu'au livret d'opéra ou à la pièce de théâtre, en passant par les adaptations, les feuilletons et les comédies de situation écrites pour des comédiens bien précis.

D'après lui, avant toute chose, le dramaturge doit posséder cinq qualités essentielles :

— il devra s'intéresser à l'humain;

- il devra avoir le sens de l'observation visuelle et auditive, combiné au don d'imagination : l'observation seule ne lui permettrait pas de dépasser le reportage;

- il devra avoir «l'instinct créateur»;

- il devra avoir «l'oreille poétique», être sensible aux effets sonores issus de l'art radiophonique. Beaucoup d'écrivains souffrent en effet d'une certaine surdité à l'égard des mots comme d'autres personnes souffrent de cécité à l'égard des couleurs;

- enfin, le dramaturge sera un conteur.

À ces cinq qualités qui, selon Wilson, composent le talent dramatique vont s'ajouter des exigences que le média télévisuel impose à l'auteur :

- le dramaturge devra connaître les limites de cette technique et apprendre à en tirer le meilleur parti possible; il devra accepter la discipline particulière qu'elle requiert;

13. Idem.

14. WILSON, Donald, «L'écrivain de T.V.», in Revue de l'Union Européenne de Radiodiffusion, cahier B, $\mathrm{n}^{\circ} 63$, septembre 1960, p. 14. 
- il devra d'autre part oublier toutes les exigences de la scène et tous les termes de métier originaires du théâtre;

- il devra ensuite penser à la télévision en termes de technologie, c'est-à-dire par rapport à sa destination finale et en fonction de l'utilisation des caméras et des micros propres à la télévision;

- si le dramaturge ne doit pas plus penser en termes de cinéma qu'en termes de théâtre, «ce qu'il doit connaître, en revanche, c'est la «grammaire» du film, tout comme quiconque veut parler couramment une langue doit en posséder les ressources», car il devra visualiser l'action et le rythme de son ouvrage et devra déterminer les moments de l'action qui seront traduits par les seules ressources de l'image sans dialogue, en concordance avec ce que lui permettent les limites des moyens techniques utilisés.

Seule une telle connaissance des restrictions ou des limites de la télévision permet d'en exploiter pleinement les ressources, et l'auteur de télévision, selon Wilson, peut alors se permettre beaucoup de libertés : le dialogue ne constitue plus toute la pièce; grâce à la mobilité extraordinaire qu'offre la succession des séquences, son ouvrage obtient une trame plus serrée; grâce aux artifices techniques et aux procédés visuels, le rythme peut devenir très rapide; enfin, sur les chaînes non commerciales, en évitant pauses et entractes, césures artificielles, l'auteur pourra situer à sa convenance les grands moments de l'action.

\section{... et au Canada (réseau français)}

Bien que la télévision ait emprunté des éléments formels au théâtre, au cinéma ou à la littérature, on ne peut écrire pour ce média comme on le fait pour le théâtre ou pour le cinéma, constate Jean Basile, écrivain et critique de télévision au journal le Devoir à Montréal.

Trois qualités, pour lui, sont nécessaires : le sens de la vie collective, le sens de l'événement et le sens du concret : une série dramatique à la télévision «est un reportage imaginaire sur des habitudes de vie d'un groupe connu, photographié sur le vif $» .^{15}$

Cependant, à la différence du théâtre, la télévision n'est pas un art en direct puisque la caméra sert d'intermédiaire en choisissant l'image et en imposant le point de vue. Au contraire du théâtre qui exige une continuité linéaire, une dramatique est enregistrée scène par scène, puis montée. Le décor et l'éclairage par leur raffinement technique et les procédés de composition de l'image offrent des avantages que ne possêde pas le théâtre.

D'autre part, la télévision diffère du cinéma du point de vue technique tout comme par la nature différente de leurs contraintes économiques.

15. BASILE, Jean, l'Écriture Radio-Télé, Montréal, Éditions Radio-Canada, 1976, p. 14. 
Enfin, pour ce qui est de l'écriture proprement dite, l'art dramatique télévisée est collectif et le script peut subir beaucoup de modifications entre le moment où il a été écrit et celui où il deviendra ouvre de télévision.

L'action doit être simple, concise, facilement compréhensible et divisée en séquences plutôt courtes, devant avoir un début, un milieu et une fin. C'est son traitement qui en fera tout l'intérêt.

Les personnages, «puisque c'est à eux que s'identifiera le spectateur», ${ }^{16}$ ne devront pas être flous ou incohérents ni trop complexes non plus. En plus d'être cohérents d'un bout à l'autre de la pièce, ils devront être identiques à eux-mêmes d'émission en émission, dans le cas d'une série.

Pour ce qui est du dialogue, il doit être en langue parlée; il doit être tributaire de l'image qui peut souvent le remplacer; les scènes d'action doivent avantageusement remplacer toute narration littéraire : l'illustration visuelle doit être préférée aux paroles.

\section{Idéologies et mode narratif}

Si nous effectuons le bilan critique de ces diverses pratiques de l'écriture télévisuelle, nous pouvons constater que nous sommes assez loin d'un «laboratoire du récit»; et si l'écriture, comme l'affirme Barthes, est «le rapport entre la création et la société», ${ }^{17}$ ce rapport est, à la source, irrémédiablement faussé. Il faut remarquer tout d'abord l'absence de l'élément acoustique qu'on peut considérer comme une partie intégrante de l'écriture ou de l'expression télévisuelle, et dans cette conversion de l'écriture à la parole visible, la musique ne servira que d'accompagnement, de fond sonore, la télévision se privant ainsi des expériences radiophoniques, en plus d'oublier la qualité du langage parlé, spontané, issu du direct. Cette corrélation du son et de l'image que propose la télévision par sa manière autre d'entendre et de voir, suscite pourtant d'importants changements de signification, de nouvelles possibilités d'espace de présence et pousse l'imagination à établir des rapports, à devenir créatrice. Micro et caméra traduisent la réalité objective des champs sonores ou visuels : «Avec un micro, on dispose (...) d'une loupe acoustique. Le micro, lentille convergente, donne d'un objet éloigné une image plus petite que lui. Pour un éloignement convenable, l'image devient égale à l'objet, puis grandit si l'objet s'en rapproche pour aboutir à une sorte d'image virtuelle, sans «localisation» précise et qui correspond aux «voix intérieures» obtenues en gros plan».18 On pourrait signaler l'absence d'intérêt dans toutes ces pratiques pour le montage visuel, le montage sonore et leur contrepoint...

16. BASILE, Jean, l'Écriture Radio-Télé, Montréal, Éditions Radio-Canada, 1976, p. 60.

17. BARTHES, Roland, le Degré zéro de l'écriture, Paris, Editions du Seuil, coll. «Points", $\mathrm{n}^{\circ} 35,1953-1972$, p. 14.

18. BERNHART, José, «De l'espace de présence dans la technique Radio-Cinéma-Télévision à la mise en scène théâtrale», in La Revue du Son, avril 1954. 
Tous ces conseils à caractère dogmatique nous apparaissent en fait comme une désignation, au moyen de signes bien clairs, d'une expression dite «télévisuelle» plus souvent absente que réelle, puisqu'ils tendent à l'édification d'un langage mythique «dont la fonction est de déformer, non de faire disparaître... Le rapport qui unit le concept du mythe au sens est essentiellement un rapport de déformation...». ${ }^{19} \mathrm{La}$ télévision devient ainsi le lieu privilégié d'une écriture «qui a pour mission de faire coïncider frauduleusement l'origine du fait et son avatar le plus lointain, en donnant à la justification de l'acte, la caution de sa réalité».20

Par la glorification d'un système de formes particulier et par l'intimidation qu'il provoque chez l'«inculte» ou chez l'«acculturé», l'édiction d'un code narratif officiel dont le but est de reproduire les formes admises, fait en sorte que la télévision devient le moyen d'expression par excellence de la culture industrialisée, en reproduisant les valeurs au pouvoir : l'auteur de télévision, devenu conteur, corrobore certes l'idée de Mc Luhan selon laquelle, grâce à l'électricité, l'électronique et leurs sous-produits, nous vivons désormais dans un village global, dans un monde qui se contracte et retrouve, à une échelle considérablement plus vaste, la cohésion tribale du premier âge. Mais d'autre part, dans la pratique, son exemple sous-entend qu'une bonne émission ne doit être rien de plus qu'une histoire bien racontée, sans complications, ne devant pas provoquer ou même rencontrer de résistance chez le téléspectateur, mais bien plutôt son adhésion. L'auteur de télévision devient ainsi scribe ou scripteur alors qu'il devrait être un auteur dans le sens complet du terme, c'est-à-dire responsable des formes qu'il créerait, devenant avec les techniciens l'équivalent du chef d'orchestre avec ses musiciens, ayant droit enfin à l'erreur, à l'impopularité même.

Entre le cinéma et la télévision, il n'y a plus de différences d'ordre technique ou stylistique, et même dans le domaine de la production, des films à grand spectacle tout comme des reportages à base de documents et d'interviews sont produits indistinctement par l'industrie cinématographique et la télévision, parfois dans les mêmes studios avec les mêmes équipes. La seule différence qui semble persister réside dans le mode de diffusion et ce qu'il implique au niveau de l'appareil critique et des réactions des spectateurs : alors que les six films d'une heure sur les Indes, réalisés par Louis Malle pour la télévision, restent pratiquement inconnus, nul n'ignore "Calcutta», son film d'une heure et demie pour le cinéma, bien que ce dernier ait été vu par moins de spectateurs ou de cinéphiles et que les émissions, tout comme le film, aient été conçus avec des matériaux de même type et montés selon des méthodes semblables.

19. BARTHES, Roland, Mythologies, Paris, Editions du Seuil, coll. Pierres Vives, 1957, p.229.

20. BARTHES, Roland, le Degré zéro de l'écriture, Éditions du Seuil, coll. «Points», $\mathrm{n}^{\circ} 35$, 1953-1972, p. 22 
L'un des effets caractéristiques de la télévision est de faire éclater les genres : mais la première réaction à cela fut d'établir un cloisonnement et une hiérarchie entre les genres d'émissions en nous faisant croire qu'ils étaient à la fois différents entre eux et nouveaux par rapport aux autres. Or, d'une part, les contraintes de l'industrialisation qui pèsent sur la fabrication des émissions tant au niveau budgétaire que formel, le traitement technique identique, et surtout l'acceptation des limitations propres à la télévision qui ne sont pas sans quelque parenté avec les pressions d'un type de censure qui procède moins par l'interdit que par l'omission et qui ne brime plus personne puisqu'elle semble être passée dans les moeurs, font que les émissions finissent par se différencier avec difficulté les unes des autres, sinon par la place qu'elles occupent au créneau horaire; d'autre part, si l'on peut dire que les types d'émissions spécifiques à la télévision sont en fait des genres narratifs distincts qui ont été transformés, trop souvent laminés par elle tout comme certains d'entre eux l'avaient été par la radio ou par le cinéma, on ne peut guère prétendre qu'elle les a engendrés : le "feuilleton", aussi appelé «télé-roman», provient de la littérature et son pays d'origine est l'Angleterre. Le premier, publié en 1719 par le London Post, n'est nul autre que le «Robinson Crusoë» de Daniel De Foë; les aventures de Sherlock Holmes sous forme de nouvelles publiées régulièrement, constituent l'une des premières «séries», et si le «quiz» vient de la radio, le théâtre populaire nous a légué l'émission de "variétés».

Enfin, comme la télévision constitue un croisement de réseaux, un carrefour à la fois de toutes les grandes disciplines de production et de diffusion et de la plupart des genres narratifs courants, la notion d'ouvre télévisée et celle d'auteur de télévision ont fait exploser les limites trop étroites auxquelles on les avait jusque-là restreintes. Cela ne devrait toutefois pas nous faire oublier que la télévision assume des fonctions sociales assez diversifiées : certaines sont évidentes comme l'information, l'éducation, la diffusion culturelle; d'autres cependant, bien qu'elles soient hautement proclamées, le sont en réalité beaucoup moins, la recherche artistique par exemple, qu'habituellement remplissent le «téléthéâtre» et la «dramatique», en somme les émissions dites de fiction. Mais si la fonction de la fiction dramatique est une fonction artistique, elle agit sur les téléspectateurs par un mode esthétique auquel une dimension idéologique est indissolublement liée.

Par ailleurs, nous l'avons vu, le pouvoir administratif à la télévision va pousser à une plus grande standardisation des émissions au moyen de codes précédemment énoncés (durée, sujet, traitement, langage, image, etc.) tendance qui va, d'une part, imposer aux ouvres produites le moule de la conformité idéologique mais qui va, d'autre part, permettre de rentabiliser le coût de production des téléfilms par une «rationalisation» du marché audio-visuel; ainsi au lieu d'une coopération entre le cinéma et la télévision dans ce qu'ils ont de spécifique, et entre les réseaux des divers pays au 
moyen d'échanges ou de co-productions, un téléfilm ou un film de cinéma pourra indistinctement passer de la télévision au cinéma et de celui-ci aux cassettes ou à la vente à l'étranger, entraînant certes un élargissement considérable du marché mais aussi une restriction des possibilités de création.

Les émissions de télévision constituent une marchandise qui voyage, un produit engageant des intérêts financiers, politiques et culturels, et cette forme nouvelle de colonisation n'est pas sans liens avec l'idée du "village planétaire» chère à Mc Luhan.

D'après une étude faite par l'UNESCO, ${ }^{21}$ seuls le Japon, la Chine, les États-Unis et l'URSS produisent eux-mêmes la quasi totalité des émissions qu'ils diffusent, et si l'Amérique du Sud importe en provenance de l'Amérique du Nord entre $20 \%$ (Argentine) et $84 \%$ (Guatemala) des émissions diffusées, en Europe, seules la France et la Grande Bretagne ont su éviter une importation massive des productions américaines : environ $50 \%$ de la production qui se répand sur l'Europe est d'origine américaine et, dans les importations des pays européens, le matériel technique américain représente entre $40 \%$ et $60 \%$ de la totalité du matériel acheté à l'extérieur.

Or fondamentalement, c'est à travers les feuilletons et les longsmétrages que s'exerce cette circulation des émissions de télévision et, avec le développement du câble et des satellites, même les législations touchant le contenu diffusé ou le protectionnisme économique au niveau de la production deviennent inopérants : tous les pays ou presque sont maintenant en voie de colonisation culturelle, et l'auteur-réalisateur va devoir défendre et illustrer son style de production télévisuelle en s'efforçant de démontrer l'impossibilité de le traduire correctement dans le style du colonisateur. D'autant plus que le rôle de l'auteur-réalisateur va apparaître comme celui de déchirer tout le système d'images, de concepts, de symboles et de signes qui aliène la conscience de l'homme, c'est-à-dire, en d'autres mots, de ne plus parler de la réalité sous couvert de l'idéologie, surtout quand les défenseurs de cette idéologie ont eu l'habileté de se baptiser révolutionnaires.

«L'idéologie, écrit Althusser, est un système de représentations : mais ces représentations n'ont la plupart du temps rien à voir avec la «conscience» : elles sont la plupart du temps des images, parfois des concepts, mais c'est avant tout comme structures qu'elles s'imposent à l'immense majorité des hommes sans passer par leur "conscience». Elles sont des objets culturels perçus-acceptés-subis et agissent fonctionnellement sur les hommes par un processus qui leur échappe. Les hommes «vivent» leur idéologie comme le cartésien "voyait» ou ne voyait pas - s'il ne la

21. «La télévision circule-t-elle à sens unique? Revue et analyse de la circulation des programmes de télévision dans le monde", in Etudes et Documents d'information, $\mathrm{n}^{\circ} 70$, Unesco, 1974. 
fixait pas - la lune à deux cents pas : nullement comme une forme de conscience, mais comme un objet de leur «monde» - comme leur «monde même». L'idéologie est alors l'expression du rapport des hommes à leur "monde», c'est-à-dire l'unité surdéterminée de leur rapport réel et de leurs rapports imaginaires à leurs conditions d'existence réelles». ${ }^{22}$

La fiction télévisuelle est surtout apparue comme étant limitée aux «feuilletons» et aux «séries» mais aussi à un niveạu différent, aux «téléthêâtres» et aux «dramatiques». Or si nous considérons la grille des émissions comme canevas d'un texte idéologique dont tous les éléments sont susceptibles d'être permutés à l'intérieur d'un même réseau, ou encore d'un réseau à l'autre, les échanges commerciaux en font foi, chaque type d'émission; chaque émission même devient fragment d'une fiction continue et, partout, véhicule de cette idéologie qui, pour certains, apparaît dans la fonction de représentation d'une émission c'est-à-dire dans l'écart créé entre l'émission et le réel, pour d'autres dans sa fonction de simulacre en considérant l'émission non plus comme représentation du réel mais comme partie intégrante de celui-ci.

Par ailleurs un certain travail va aussi se faire sur le mode narratif utilisé dans les productions à caractère créatif pour la télévision. Alain Robbe-Grillet proposait une manière de classifier les films de cinéma par leur mode narratif ${ }^{23}$ qui pourrait tout aussi bien convenir pour les films de télévision : «Il faut appeler «film narratif» tout film qui se conforme par principe au code de la narration en place. Il s'agit donc là d'un cinéma de reproduction : qui se contente de reproduire les formes reçues et qui constitue un des grands organes grâce auxquels les valeurs au pouvoir se reproduisent. C'est lui qu'on enseigne dans les écoles, lui que dans leur écrasante majorité les réalisateurs s'efforcent de mettre en pratique, lui que les critiques traditionnels définissent par leur sentence favorite : un bon film, c'est une belle histoire bien racontée».

À l'opposé, Robbe-Grillet définit comme «film non-narratif» celui où les effets de narration sont totalement absents, ou du moins n'occupent que des temps extrêmement brefs, et le reproche qu'il lui adresse est de ne pas être programmable dans les circuits de distribution normaux : on ne peut lutter contre l'idéologie que de l'intérieur même, dit-il, quiconque croit en sortir se condamne au silence.

Entre le narratif et le non-narratif, il définit un troisième type de cinéma qu'il appelle «dys-narratif», qui détruit de l'intérieur l'idéologie en place. Toute une thématique, une qualité de photographie, une panoplie d'objets, d'acteurs, de situations dramatiques, etc. s'y retrouvent et ce type de cinéma «vise à introduire dans le système des dysfonctionnements qui le

22. ALTHUSSER, Louis, in Pour Marx, Paris, Maspero, 1966, pp. 239-240.

23. ROBBE-GRILLET, Alain, "L'argent et l'idéologie», in le Monde, 26 février 1976, p. 17. 
font grincer, soit qu'il monte dans un autre ordre des fragments découpés dans le discours régnant et empêche ainsi l'effort totalitaire de continuité (l'idéologie ne peut fonctionner que comme un tout, clos et continu), soit qu'il en désigne les effets illusionnistes par un renvoi du matériau et de la technique à leur statut d'artifices (l'idéologie ne s'impose que sous le masque du naturel), soit qu'il en retourne comme un gant les moteurs et les mécanismes (l'idéologie ne travaille bien que si on ne la voit pas travailler)».

C'est évidemment dans la télévision de type non-narratif que va s'épanouir l'art-vidéo, mais c'est plutôt du côté d'une production de type dys-narratif (dont un bon exemple serait l'œuvre télévisuelle d'Hubert Aquin) que va tenter de s'affranchir une certaine forme d'utilisation de la télévision; car si l'idéologie est l'art de donner une cohérence apparente aux contradictions d'un système, la fiction télévisée, à travers les pouvoirs du discours narratif certes, mais aussi à travers la vision du monde qu'elle reflète et la relation du spectateur aux personnages, n'est rien d'autre que l'instrument de cette idéologie.

\section{Le spectacle télévisuel : art ou artisanat?}

En assimilant la télévision aux «arts de reproduction» par un rapprochement avec la photographie et le cinéma ou du moins avec une certaine conception de ceux-ci, on reconnaît les rapports étroits qui la lient au réel qu'elle feint d'imiter, le déguisant, évacuant tout effort d'invention par l'imitation de modèles consacrés, et rendant le sens de toute ouvre, s'il est encore possible de parler d'œuvre, tellement déterminé et univoque, que toute polysémie devient impossible. Cette approche laisserait dans l'ombre toute une gamme d'activités propres à la télévision.

Pourrait-on alors parler avec Tardieu d'un «art de diffusion» tout comme pour la radio? En effet l'objectif des émissions de télévision se situe entre deux pôles : capter, non plus le mouvement mais l'événement, et le diffuser; d'autre part, produire des "oeuvres d'art» pour en effectuer la diffusion. Or la télévision ne semble pas pouvoir intégrer une recherche proprement formelle, la forme ayant elle-même une fonction critique, ou une production qui inscrit en elle la production comme élément essentiel, ne renvoyant qu'à son dynamisme fondamental : de quelles œuvres d'art s'agit-il donc? Les prétendues "œuvres d'art» diffusées par la télévision se rapprochent beaucoup moins du refus que signifie la production d'une œuvre véritable, que du «non-art» selon Marcuse, c'est-à-dire du renoncement à l'art.

Se rapprocheraient-elles enfin de ce que Mikel Dufrenne, à la suite de Théodor Adorno, définit par «l'art de masse» dans la société actuelle, art produit pour le peuple considéré comme masse par des gens qui se nomment encore des artistes? Art essentiellement conservateur, paternaliste et divertissant, répondant aux besoins et aux attentes déterminés de la masse 
(par les sondages ou les préjugés), et dont l'une des fonctions, nous dit-il, est de faire valoir, par contraste, «l'art véritable», «la culture», en somme l'art de l'élite : «accepter l'idée d'un art de masse qu'on tient en même temps pour médiocre, c'est rehausser, selon que l'exige l'idéologie des dominants, l'affirmation à la fois d'une élite, à laquelle on s'intègre par là même, et d'un autre art qu'on tient du même coup pour majeur et dont on ne met pas en question l'authenticité, ni l'institutionnalisation». ${ }^{24}$

De son côté, Adorno fait remarquer que «tout art dont l'objectif est d'occuper les loisirs est devenu un passe-temps et récupère simultanément les contenus et les formes de l'art autonome traditionnel, considérés comme des «biens culturels». C'est précisément ce processus de l'amalgame qui détruit toute autonomie esthétique : ce qui est arrivé à la sonate $A u$ clair de lune, chantée par un chour et jouée par un orchestre d'ambiance, est en définitive ce qui arrive à toute chose. Quant à l'art qui ne se soumet pas, il est complètement écarté de la consommation et condamné à l'isolement. Tout le reste est démonté, dépouillé de sa signification, puis remonté. Le seul critère déterminant de cette procédure est d'atteindre le consommateur de la manière la plus efficace possible».25

En réalité, la production télévisuelle, nivelée et pré-digérée, oblitérée en quelque sorte par le média, se rapprocherait assez de cet art manipulé, de cet art de consommation qui s'adresse au goût et à la réceptivité standardisés, à l'imagination et à la créativité presque annihilées. Dans ce cas, il est vrai que l'on peut parler de la télévision comme d'un art, et mentionner que l'aspect technique du moyen de production en a déterminé l'aspect esthétique de manière spécifique...

Sans affirmer pour autant que la technicisation de l'œuvre mène en définitive à la liquidation de l'art, il nous faut tout de même admettre que le conflit suscité par la télévision considérée comme un art, est double, puisqu'il nous oblige à reconsidérer à la fois la notion d'art et, par le biais du récepteur, la notion d'œuvre. L'art moderne remet profondément en cause la signification coutumière de la notion d'art. Ce dernier, jusqu'ici produit en fonction de sa consommation, est maintenant considéré comme objet de réception, déchargeant le spectateur de toute activité. Cela signifie que le récepteur, systématiquement transformé, est impliqué dans la réalisation même de ce qui lui est soumis, et de cette inclusion dans le processus de production va découler, pour l'auteur, la perte de son statut messianique, et pour le récepteur, lecteur, auditeur ou spectateur, la nécessité de se mettre «dans l'état ou mieux dans l'activité d'un

24. DUFRESNE, Mikel, «L'art de masse n'existe pas», in Revue d'esthétique, nos $\mathbf{3 - 4}$, Union générale d'Édition, coll. 10/18, Paris, 1974, p. 18.

25. ADORNO, Théodor W., in Musique de Cinéma, Éditions L'Arche, coll. Travaux, $\mathrm{n}^{\circ} 17$, Paris, 1972, p. 10. 
performateur, qui sait déplacer, grouper, combiner, agencer, en un mot (s'il n'est pas trop usé) : structurer (ce qui est bien différent de construire ou reconstruire, au sens classique)». La lecture de l'ouvre moderne va consister à «l'écrire de nouveau, à traverser son écriture d'une nouvelle inscription... (à) l'attirer dans une praxis inconnue», nous dit Barthes. ${ }^{26}$

Malheureusement, si l'on considère la télévision en regard de ces approches nouvelles et des rapports nouveaux qu'elles suscitent, l'art télévisuel, tel du moins qu'on pourrait le postuler, risque de se réduire aux constatations de Godard pour qui, à la télévision, il n'y a pas d'œuvres, il n'y a que des heures...

Pour hâter l'avènement d'un art télévisuel véritable, il importerait, d'un point de vue pratique, d'avoir une volonté de reconsidérer tout le rapport communicationnel existant.

Ainsi, comment ne pas. s'étonner de l'absence de tout programme d'action pédagogique lié à ce fait de civilisation, visant à donner aux jeunes téléspectateurs une connaissance pratique du processus, couplée à l'acquisition d'outils critiques face à une utilisation immédiate mais aussi face à une profonde modification de toute notre conception de la réalité. À commencer par la lecture : ne sommes-nous pas, devant l'image, de véritables analphabètes à côté d'enfants à qui l'on veut montrer la culture de l'imprimé?

Comment ne pas s'étonner de l'absence d'alternatives visant à faire mentir cette certitude de Baudrillard que les gens ne se parlent plus, qu'ils sont définitivement isolés face à une parole sans réponse lorsqu'ils sont devant le petit écran : ce n'est pas en étant réfractaire à la recherche, à l'expérimentation mais aussi à l'effort individuel, que surgiront des formes de création et de diffusion originales : les arts du spectacle plus traditionnels, fort heureusement, n'ont pas délaissé cette espèce de dynamisme intérieur qui leur est propre! L'apport du direct avait, avec une approche nouvelle du travail d'équipe, fait l'originalité de la forme télévisuelle : si des émissions intéressantes sont conçues en équipe, certaines destinées à la jeunesse entre autres, le direct est tellement stéréotypé qu'il s'est affranchi depuis longtemps de toute imagination...

Enfin du côté de la formation à la création télévisuelle, comment ne pas se rendre compte que la télévision n'existe déjà plus; qu'en ce domaine, la créativité va s'exercer et s'entretenir au moyen de l'informatique couplée à l'écran vidéo; que le bouleversement des modes de création va nécessiter l'apprentissage de disciplines nouvelles : les graphistes et les musiciens ont déjà compris cela en terme d'affranchissement, tandis que l'auteur apprivoise encorè le typewriter (machine à écrire/machine à imprimer)!

26. BARTHES, Roland, "Musica Practica», in l'Arc, no 40, numéro sur Beethoven, Aix-enProvence, ler trimestre, 1970, p. 17. 
Tout se résumerait-il alors à une question d'attitude? Entre l'homme et l'outil, notre choix se portera-t-il vers une synthèse originale, une solution liée à de nouveaux paradigmes, ou plus simplement vers l'indifférence ou encore, la béate fascination? 